\title{
Phase Modulated Radio-over-Fiber for Efficient 5G Fronthaul Uplink
}

\author{
Emine Moutaly, Philippos Assimakopoulos, Member, IEEE, Shabnam Noor, Member, IEEE, Salim \\ Faci, Senior Member, IEEE, Anne-Laure Billabert, Nathan J. Gomes, Senior Member, IEEE, \\ Mohamed Lemine Diakité, Colm Browning, Member, IEEE and Catherine Algani
}

\begin{abstract}
Analog radio-over-fiber technology is gaining interest as a potential candidate for radio signal transport over the future fronthaul section of the $5^{\text {th }}$ generation (and beyond) radio access network. In this paper, we propose a radio-over-fiber fronthaul with intensity modulation in the downlink and phase modulation with interferometric detection in the uplink, for simplified and power efficient remote units. We conduct an experimental investigation and verification of theoretical and simulation models of the performance of the phase-modulated uplink and demonstrate the ability of such an architecture to transport single-channel and multi-channel 5G-type radio waveforms. Experimentally verified data rates of $4.3 \mathrm{Gbps}$ and simulation-based predictions, using a well matched-tomeasurements model of the uplink, of 12.4 Gbps are presented, with error-vector magnitude performance well within relevant standard specifications for 64-QAM.
\end{abstract}

Index Terms-5G network, radio-over-fiber, fronthaul, phase modulation.

\section{INTRODUCTION}

$\mathrm{T}$ he $5^{\text {th }}$ generation (5G) Radio Access Network (RAN) will have to support high data rates, required for enhanced Mobile Broadband (eMBB) services, low latencies for ultrareliable low-latency communication (URLLC) services and centralized processing [1]. To this end, the 3rd Generation Partnership Project (3GPP) standard Release 15 has defined the main characteristics of the 5G RAN architecture [2]. A functional split has been proposed [3], with the aim of reducing the amount of traffic carried over the fronthaul link compared to traditional digitized radio transport techniques, such as those employed by the Common Public Radio Interface (CPRI) industry specification [4]. As shown in Fig. 1, the 5G base station (gNB) is divided into 3 units: Central Unit (CU), Distributed Unit (DU) and Remote Unit (RU) [2]. In a standalone configuration with independent RU, DU and CU locations, the option 2 split, shown in the lower part of Fig.1, is already defined for the F1 interface between $\mathrm{CU}$ and DU, but

This work was supported in part by the European Cooperation for Science and Technology under Grant CA16220. Philippos Assimakopoulos acknowledges support by the EU Horizon 2020 5G-DRIVE project (grant agreement no. 814956).

(Corresponding author: Emine Moutaly.)

E. Moutaly, S. Faci, A-L. Billabert and C. Algani are with ESYCOM (FRE2028), CNAM, CNRS, ESIEE Paris, Université Paris-Est Marne-laVallée, 292 rue Saint-Martin 75141 Paris Cedex 3, France (e-mail: moutally@gmail.com; salim.faci@lecnam.net; annelaure.billabert@lecnam.net; catherine.algani@lecnam.net). the F2 interface between RU and $\mathrm{CU}$ is still under investigation. The range of candidate split points for the F2 interface is indicated in Fig. 1. Depending on the final split point choice, the data rate required over this segment will certainly be reduced, but at the cost of more complex RUs (that is, RUs will require more processing functions compared to traditional radio heads employing a CPRI-type split). Even with an intra-PHY split, data rate requirements over the F2 interface, magnified by multiple antenna techniques such as massive Multiple-Input and Multiple-Output (mMIMO) and 5G bandwidths (up to 400 $\mathrm{MHz}$ ) will still impose significant demands on the transport network [3], [5].

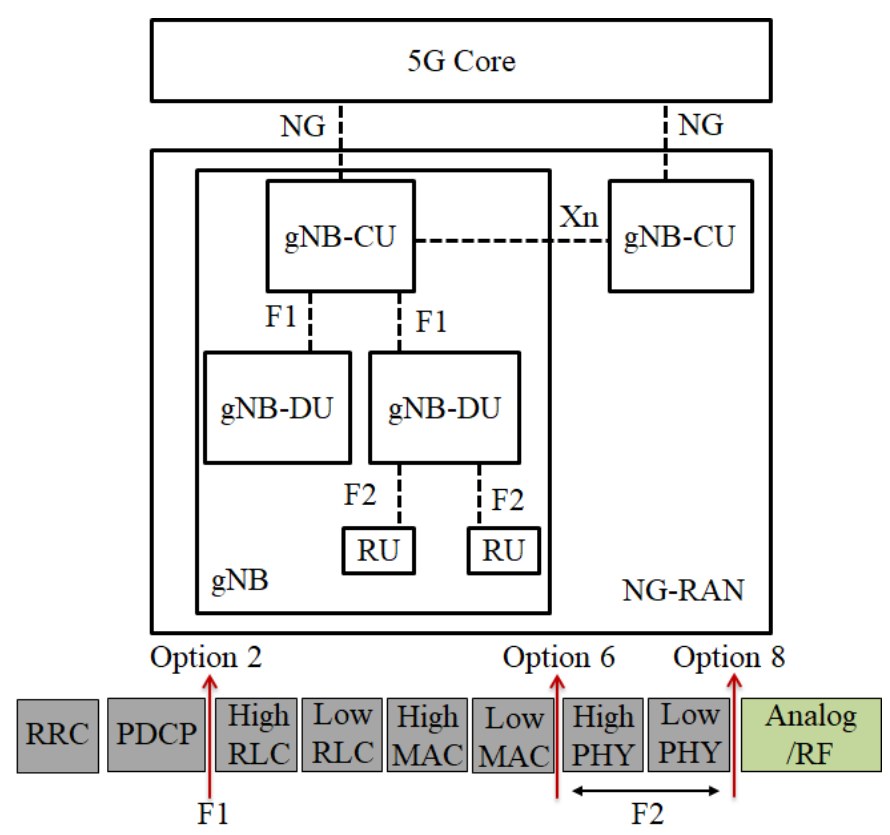

Fig. 1. 5G NG-RAN architecture and the different split interfaces, including candidate split points (adapted from [2]).

P. Assimakopoulos, S. Noor and N.J. Gomes are with the Communications Research Group, University of Kent, Canterbury CT2 7NT, U.K. (e-mail: p.asimakopoulos@kent.ac.uk; s.noor@kent.ac.uk; n.j.gomes@kent.ac.uk).

C. Browning is with the School of Electronic Engineering, Dublin City University, D09W6Y4 Dublin, Ireland (e-mail: colm.browning@dcu.ie).

M. Diakité is with Computer Science Department, University of Nouakchott Alaasrya (e-mail: diakite@una.mr) 
Furthermore, split points closer to the 5G MAC/PHY interface will impose restrictions in the deployment of cooperative base stations, as in distributed MIMO scenarios [3], [5]. For these reasons, analog transport through Radio-overFiber (RoF) techniques has recently gained renewed interest as an alternative to such split interfaces [6-8]. As an example, using CPRI (Option 8), for $8 \times 8 \mathrm{MIMO}$ and $100 \mathrm{MHz} 5 \mathrm{G}$ signals, the projected line rate is approximately $49.152 \mathrm{Gbps}$. Under certain assumptions, this rate can be reduced by an order of magnitude with a functional split at the MAC/PHY (Option 6) interface [6]. In an equivalent analog system, a much higher spectral efficiency can be achieved [8], for example, a bandwidth somewhat larger than $800 \mathrm{MHz}$ would be required (theoretically). In general, both the signal bandwidth (including aggregate bandwidth if multiple signals are transported) and the carrier frequency have to be taken into account when comparing analog and digitized RoF techniques [9]. However, the requirements imposed to the transport architecture by the choice of carrier frequency, can be relaxed through remote delivery (of the local oscillator signal) by employing optical heterodyning approaches [10], [11] or low complexity electrical up-conversion approaches [12], [13]. A number of analog transport schemes have been investigated for the transmission of OFDM-type wideband signals [6-17]. At the same time, there has been a shift towards the millimeter-wave $(\mathrm{mmW})$ region of the radio frequency spectrum due to increased demand for wireless bandwidth. Traditional RoF-Subcarrier Multiplexing (SCM) schemes, for sub $6 \mathrm{GHz}$ [14], [15] or mmW frequencies [13], rely on analog components to multiplex signals and thus lack scalability (in terms of cost and complexity) for wide bandwidth channels and large multiplexes. Recent work on digital multiplexing techniques (with analog transport) are promising [10], [12], [16], [17], but do face a number of limitations especially with regards to complexity, processing overheads and sampling rates, although a number of solutions have been proposed [10], [16]. Furthermore, the next generation RAN is placing new implementation constraints, requiring techniques that are as flexible and scalable as possible [10].

Most proposed schemes (for example most of the aforementioned works) for RoF applications suggest the use of Intensity Modulation-Direct Detection (IM-DD), either with direct or external modulation, as both are certainly mature technologies for Radio Frequency (RF) signal transport [14], [18]. Direct modulation suffers from RF modulation-induced chirp effects. The use of external modulators powered by a continuous wave laser, and most commonly employing a MachZehnder Modulator (MZM), is the alternative solution. While both MZM-based and direct modulation links suffer from nonlinear distortion (static, from the modulator transfer characteristic for MZM links, but both static and dynamic for laser modulation), MZM links offer improved performance in terms of resilience to chromatic dispersion by avoiding laser chirp, and in terms of higher link RF gain, if the received optical power is high [18].

An improvement to these limiting characteristics is offered by phase-modulated RoF links. In [19], the authors have demonstrated a gain enhancement of $12 \mathrm{~dB}$, a noise figure reduction of $9 \mathrm{~dB}$ and a dynamic range improvement of $2 \mathrm{~dB}$ for a Phase-Modulated link with Interferometric Detection (PM-ID) compared to an intensity modulated link with the same RF signal and laser characteristics. Another architecture based on phase-modulation with digital coherent detection that is suitable for mmW systems has been proposed in [20]. This architecture, despite demonstrating good performance, requires an efficient and complex digital signal processing block for demodulation.

Simplification of RUs in terms of cost and complexity is of major importance, especially for high-density cell deployments in the future $5 \mathrm{G}$ (and beyond) networks. For this, we propose an optimized RoF for $5 \mathrm{G}$ fronthaul based on phase-modulation with interferometric detection for the uplink. The use of phase modulation in the uplink contributes to the improvement of the energy efficiency of the RUs, as no electrical bias is required for the phase modulator and they can be laser free. Furthermore, the optical wavelength for the uplink can be centrally controlled and distributed from the DU, offering centralization gains. For the downlink, a classical MZM-based RoF topology can be employed. This architecture, built with low-cost photonic components, can provide adequate performance for sub- $6 \mathrm{GHz}$ radio frequencies. For higher frequencies such as $\mathrm{mmW}$ bands beyond $28 \mathrm{GHz}$, an SCM/Intermediate Frequency (IF) RoF (SCM/IF-RoF) scheme, using the same architecture presented here can be employed. Down-conversion can be achieved either through an electrical down-conversion block at the RU [12], [13] or alternatively through a remotely delivered carrier, generated by an optical heterodyning technique [10], [11].

In this paper, we present a comprehensive model for the PMID uplink and demonstrate very good matching with experimentally measured performance for both single and multi-channel transmission. We further demonstrate for the first time, to the best of our knowledge, the ability of this type of link to transport high bandwidth, standard (CP-OFDM) 5Gtype and 5G-candidate waveforms, with both single and multichannel transmission, with performance well within 3GPP specifications.

The paper is organized as follows: In Section II we briefly describe the proposed architecture, while in Section III we first describe the modelling process of the link used for system simulations, and then present the experimental and simulation characterization used to determine analog performance metrics. In Section IV, we compare experimental and simulation results for the transmission of single and multi-channel 5G-type waveforms over the link. The paper is concluded in Section V.

\section{ARCHITECTURE DESCRIPTION}

When phase modulation of an optical signal is used for the uplink, the RF signal modulates the phase of the carrier making direct quadratic detection with photodiodes (PDs) unusable for demodulating the signal. Many approaches have been considered for the detection of the phase modulated signal [21]. Among the many approaches considered for the detection of the phase modulated signal, interferometric detection, which is based on a phase to amplitude conversion followed by a quadratic detection, is a common method [19], [22]. The phase 
to amplitude conversion is a phase discrimination operation that can be implemented using a Mach-Zehnder Interferometer (MZI) followed by a single PD or by balanced photo-detection. A more sophisticated approach to recover the phase information is to employ coherent detection which requires the use of a second laser source. The resulting beat signal is processed in the digital domain with dedicated electronics to extract the modulation signal [23]. In this work we consider interferometric detection which constitutes a low complexity alternative to other techniques.

Fig. 2 shows the proposed architecture. The downlink comprises of a traditional external IM-DD link with the incorporation of a remotely delivered optical carrier through an optical multiplexer. The optical carrier is de-multiplexed at the RU where it is phase modulated using an unbiased Phase Modulator (PM). The modulated optical signal is transported through an optical link and received by the DU where an MZI provides interferometric phase to amplitude conversion before balanced photo-detection. In this paper, only simple, unbalanced photo-detection was used in the experimental setup due to component unavailability.

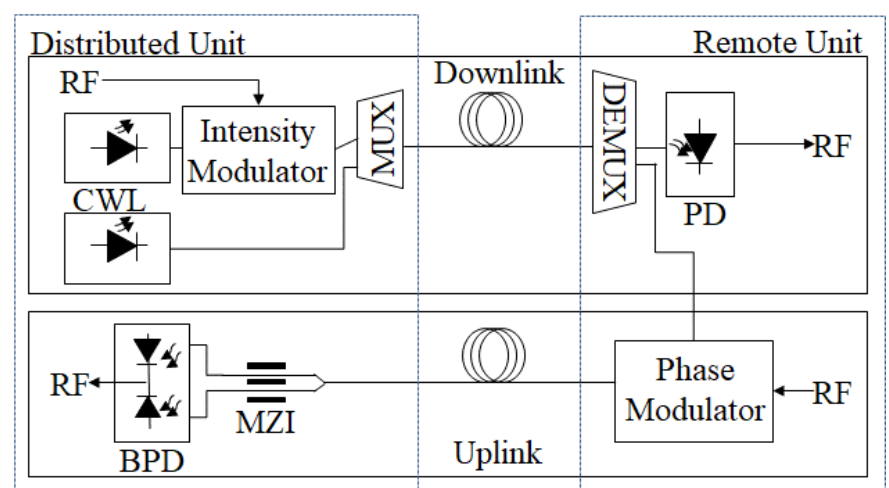

Fig. 2. Proposed 5G radio-over-fiber fronthaul. CWL, Continuous Wave Laser, MUX, Multiplexer; RF, Radio Frequency; BPD, Balanced Photodiode.

\section{RoF LINK MODELLING AND CHARACTERIZATION}

\section{A. RoF link modelling}

The RoF link is modelled with electrical equivalent circuits. In order to take advantage of analysis tools available in electrical simulators, we have opted for equivalent circuit modelling to evaluate the entire system. Circuit modelling is an efficient way of representing microwave-photonic components by transforming physical equations governing the internal component dynamics to current/voltage equations and solving them using Kirchhoff's laws [24]. These physical parameters are optimized through component measurement characterizations so that a good match between model and measured behaviors can be achieved. In some cases, where the analytical transformation of component physical equations is difficult, a transfer function representation is used instead. Device non-linearity has been taken into account in component modeling except for that of optical fiber (for the RF frequencies, optical powers and fiber lengths employed here, the effects are expected to be negligible). The optical powers used in this work are below the saturation threshold of the devices used, while the bandwidth of the PM used is much higher than the employed RF frequencies.

The modelled Continuous Wave (CW) laser source is an NP Photonics Scorpion SMPF-2030 fiber laser. The output current of the laser model represents the electrical field of the optical beam, which includes phase information. Phase and intensity noises are represented by two current noise sources with their power spectral densities related to linewidth and laser Relative Intensity Noise (RIN), respectively. The optical spectrum obtained with envelope simulation is presented in Fig. 3 with intensity and phase noise, and when noise sources are switched off.

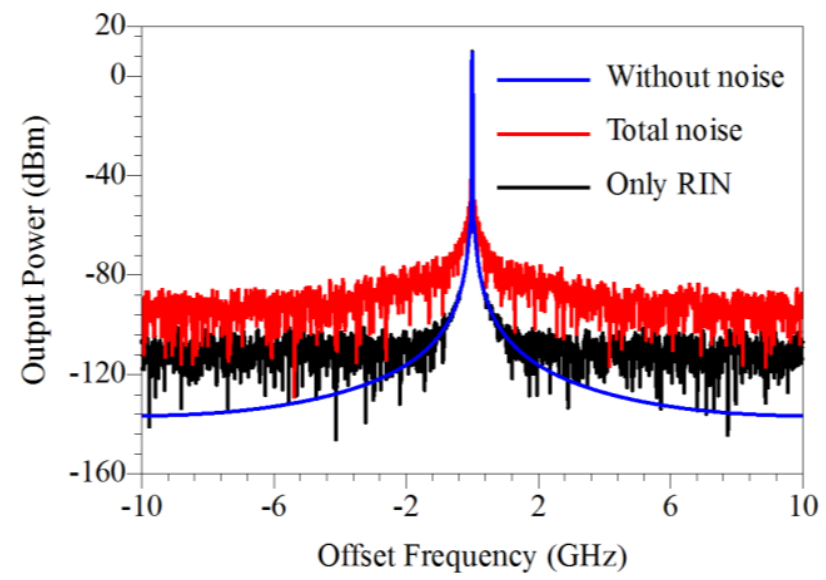

Fig. 3. Modeled output spectrum of NP Photonics Scorpion laser. Optical input power is $10 \mathrm{dBm}$.

For the PM, optical fiber and PD, we have adopted the electrical models presented in [25]. The PM is simply modelled by the built-in PM model provided in the simulator library. The electrical field at the PM output is given by

$$
E_{p h}(t)=\sqrt{\alpha_{p h} P_{i n}} e^{i\left(\omega_{o p t} t+\phi_{R F}(t)\right)},
$$

where $\alpha_{p h}$ is the input optical loss, $P_{\text {in }}$ is the input optical power, $\omega_{\text {opt }}$ is the optical angular frequency and $\phi_{R F}(t)$ is the RF modulating signal and is given by

$$
\phi_{R F}=\frac{\pi V_{R F}(t)}{V_{\pi}} \sin \left(\omega_{R F} t\right),
$$

where $V_{\pi}$ is the voltage inducing a phase shift of $\pi, V_{R F}(t)$ is the signal magnitude and $\omega_{R F}$ is the RF angular frequency.

Then, a free space MZI with a relative time delay $\tau$ in one arm and 50/50 couplers is assumed. Output electrical fields are expressed using the transfer function of the MZI, given by

$\left[\begin{array}{l}E_{1}(t) \\ E_{2}(t)\end{array}\right]=\frac{1}{2} \sqrt{\alpha_{m z i} g_{o p t}}\left[\begin{array}{ll}1 & i \\ i & 1\end{array}\right]\left[\begin{array}{cc}D(\tau) & 0 \\ 0 & 1\end{array}\right]\left[\begin{array}{ll}1 & i \\ i & 1\end{array}\right]\left[\begin{array}{c}E_{p h}(t) \\ 0\end{array}\right],(3)$

where $\alpha_{m z i}$ is the MZI optical loss, $g_{\text {opt }}$ is the optical gain and $D(\tau)$ is a delay operator. 
A symbolically defined device combined with built-in RF splitters is used to implement the MZI transfer function [25].

\section{B. Link characteristics}

The main performance metrics generally considered for RF systems analysis are link gain, $1 \mathrm{~dB}$ gain compression point, Spurious-Free Dynamic Range (SFDR) and noise figure. The detailed mathematical expressions of phase modulation-based RoF links are provided in [26]. The small-signal gain at the quadrature point of operation is approximated as

$$
g=4 \pi^{2}\left(\frac{\alpha_{p h \alpha_{f}} \alpha_{m z i} R_{p} P_{i n}}{V_{\pi}}\right)^{2} \sin ^{2}\left(\frac{\omega_{R F} \tau}{2}\right) R_{\text {in }} R_{o u t}
$$

where $\alpha_{\mathrm{f}}$ is the fiber attenuation, $R_{p}$ is the responsivity of the $\mathrm{PD}, R_{\text {in }}$ is the input impedance of the PM and $R_{\text {out }}$ is the photodetector load impedance.

The input power at the $1 \mathrm{~dB}$ gain compression point is expressed at a maximum gain (RF frequency at the half-FSR point) by

$$
P_{R F i n-1 d B}=\frac{1}{2 R_{\text {in }}}\left(\frac{0.475 V_{\pi}}{\pi \sin \left(\frac{\omega_{o p t} \tau}{2}\right)}\right)^{2}
$$

Note that (5) is the generalized expression and is not specific to the quadrature point of operation (where the sinusoidal term is equal to unity). The maximum SFDR of the link is given by

$$
S F D R_{\max }=\left(\frac{V_{\pi}{ }^{2}}{\pi^{2} R_{i n} k_{B} T B \sin \left(\frac{\omega_{o p t} \tau}{2}\right)}\right)^{2 / 3},
$$

where $k_{B}$ is the Boltzmann constant, $T$ is the temperature and $B$ is the noise measurement bandwidth. The total noise power spectral density includes thermal noise, shot noise, RIN and phase noise converted to intensity noise. Phase noise to intensity noise conversion can largely dominate other noise sources in the phase modulated link. It can be expressed as

$$
N_{\varphi-I}=\frac{\Delta v e^{-2 \pi \tau \Delta v}}{\pi\left(\Delta v^{2}+f_{R F}^{2}\right)}\left[\cosh (2 \pi \tau \Delta v)-\cos \left(2 \pi f_{R F} \tau\right)\right]
$$

where $\Delta v$ is the laser linewidth.

Thus, assuming the same link parameters, the noise level in PM-DD links depends on laser linewidth and can be higher than the noise level in IM-DD links (this issue will be discussed in more detail in Subsection III.D).

\section{Experimental setup}

The CW Scorpion fiber laser provides an optical power of 10 $\mathrm{dBm}$ at a wavelength of $1556.016 \mathrm{~nm}$ to a $40 \mathrm{GHz}$ Sumitomo PM (ref. T.PM1-5-40). A Vector Signal Generator (VSG) is used to generate the RF signal. The MZI is a WT-MINT-M-U from Kylia where one output is connected to an Erbium-Doped
Fiber Amplifier (EDFA) and the second one to an Optical Spectrum Analyzer (OSA) for monitoring. The EDFA is employed as a fixed gain amplifier. A 4.3 GHz bandwidth Appointech high responsivity PD detects the EDFA output. The amplified photo-detected signal is observed at an Electrical Spectrum Analyzer (ESA) for power measurement or with a vector Network Analyzer (VNA) for small-signal gain measurement. As the standard fronthaul length for $5 \mathrm{G}$ applications is generally less than $20 \mathrm{~km}$, the maximum loss expected coming from the optical fiber is around $4 \mathrm{~dB}$ at a wavelength around $1550 \mathrm{~nm}$. Although chromatic dispersion is included in the fiber model, its effect is negligible for the length of fiber and frequencies employed in this work [25]. Note that fronthauling applications will generally require short-reach fiber links $(<20 \mathrm{~km})$ while IF-RoF techniques can be used to allow transport of $\mathrm{mmW}$ signals at lower RF frequencies.

\section{Obtained results and discussion}

The measured and simulated small-signal gain versus RF frequency are presented in Fig. 4 and show a good agreement (note that for these measurements, the $4.3 \mathrm{GHz}$ PD was replaced by a $40 \mathrm{GHz} \mathrm{PD})$. For the simulation setup, the PM $V_{\pi}$ was set to $3.7 \mathrm{~V}$, the $\alpha_{m z i}$ to $3 \mathrm{~dB}$, the $g_{\text {opt }}$ to $30 \mathrm{~dB}$ and the MZI delay time to $166.66 \mathrm{ps}$, corresponding to an FSR of $6 \mathrm{GHZ}$. The simulation parameters are summarized in Table I.

TABLE I

SUMMARIZED SIMULATION PARAMETERS

\begin{tabular}{lc}
\hline \multicolumn{1}{c}{ Parameter } & value \\
\hline Wavelength $(\mathrm{nm})$ & 1556.016 \\
Phase modulator $\mathrm{V}_{\pi}(\mathrm{V})$ & 3.7 \\
RF frequency $(\mathrm{GHz})$ & 2 \\
Phase modulator FSR $(\mathrm{GHz})$ & 6 \\
PM input impedance $(\mathrm{Ohm})$ & 50 \\
Laser diode linewidth $(\mathrm{kHz})$ & 10 \\
Photodiode responsivity $(\mathrm{A} / \mathrm{W})$ & 1 \\
Laser RIN $(\mathrm{dB} / \mathrm{Hz})$ & -170 \\
Photodiode bandwidth $(\mathrm{GHz})$ & 4.3 \\
EDFA gain $(\mathrm{dB})$ & 30 \\
\hline \hline
\end{tabular}

From (4), the computation of the peak gain at frequency multiples of $3 \mathrm{GHz}$ corresponding to half-FSR gives a value of $-28 \mathrm{~dB}$. The gain peaks are observed at odd multiples of halfFSR while minima are observed at even multiples. These theoretical values are in good agreement with both measurement and simulation data. The RF frequency can be adjusted to the maximum point of the gain curve by adjusting the FSR. We can notice from (4) that a higher FSR provides a higher $3 \mathrm{~dB}$ bandwidth around the maximum RF gain frequency point which is equal to half-FSR. This higher FSR can be achieved by adjusting the time delay parameter ( $\tau$ in (4)) of the MZI to a smaller value.

Fig. 5 represents the measured and simulated RF gain versus input RF power for an RF signal frequency of 2 and $3.3 \mathrm{GHz}$. An RF signal at the desired frequency is provided by the VSG to the PM. The received RF power is measured with an ESA. Simulations are performed with $R_{\text {in }}$ equal to $50 \Omega$ and a matched load. According to (5), the input power at $1 \mathrm{~dB}$ gain compression is $7.12 \mathrm{dBm}$ while the simulated value at $2 \mathrm{GHz}$ is 
around $7.3 \mathrm{dBm}$. The measured value is estimated at $6.9 \mathrm{dBm}$ which is close to the values obtained through simulation and theoretical computation. The small difference in these measurements can be attributed to an inaccurate estimation of the $V_{\pi}$ value of the PM used in simulation. The additional measurement performed at a frequency of $3.3 \mathrm{GHz}$ resulted in the same $1 \mathrm{~dB}$ input compression point value, confirming the prediction from (5) that the compression point does not depend on RF frequency when the MZI is operated at the quadrature point.

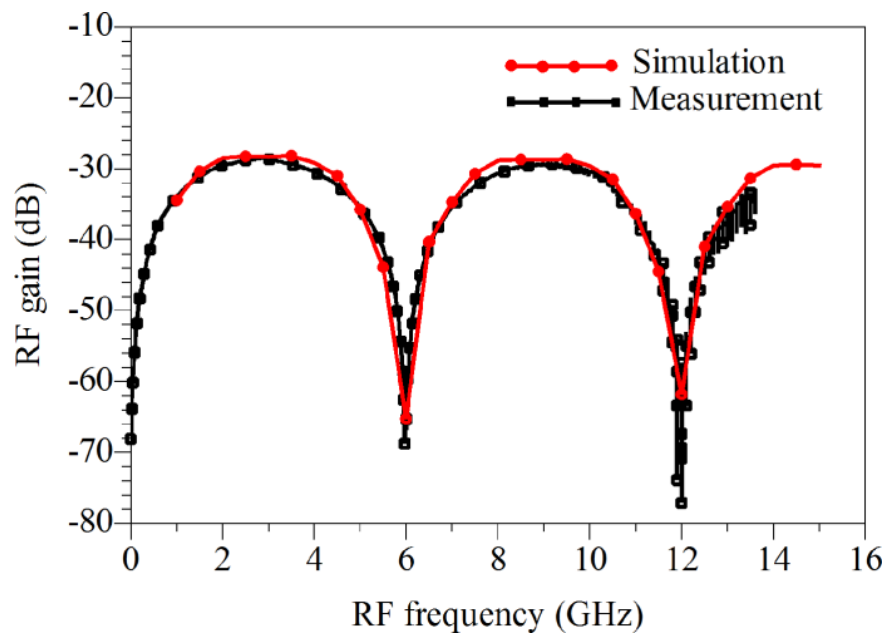

Fig. 4. Small-signal gain vs. RF frequency, measured with a VNA at $-10 \mathrm{dBm}$ input power and $6 \mathrm{GHz}$ FSR.

To evaluate the SFDR, the input third order intercept point (IIP3) of the link was measured. Two VSGs providing RF signals at 2 and $2.05 \mathrm{GHz}$ were combined at the RF input port of the PM. Output powers of the fundamental and third order mixing components were captured by an ESA and compared to simulation results as shown in Fig. 6. The simulated IIP3 value is $21 \mathrm{dBm}$ and the measured value is approximately $20 \mathrm{dBm}$. The noise power spectral density measured at the output of the link was $-157 \mathrm{dBm} / \mathrm{Hz}$. This leads to measured and simulated SFDR values of 100.4 and $102.5 \mathrm{~dB} \cdot \mathrm{Hz}^{2 / 3}$, respectively.

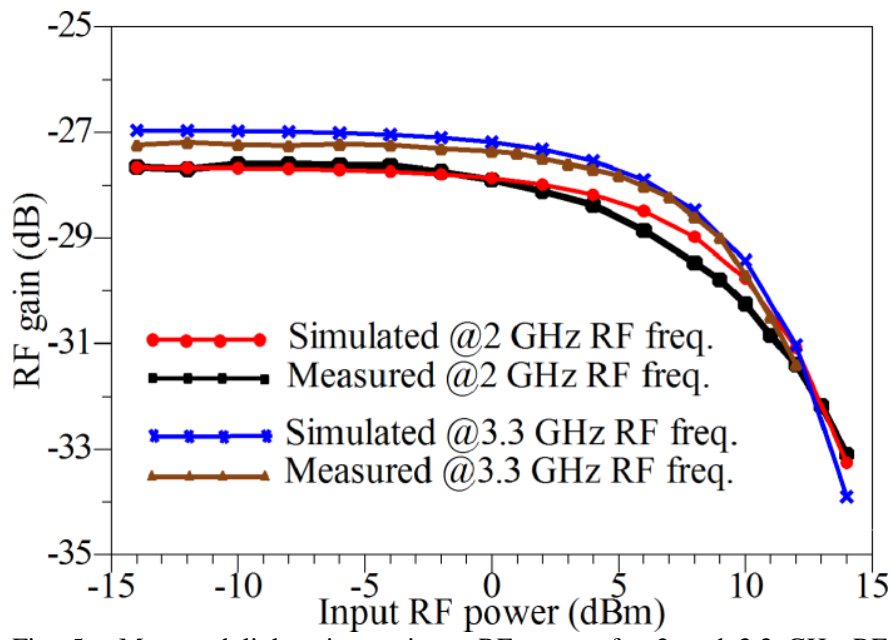

Fig. 5. Measured link gain vs. input RF power for 2 and $3.3 \mathrm{GHz} \mathrm{RF}$ frequencies and an FSR of $6 \mathrm{GHz}$.

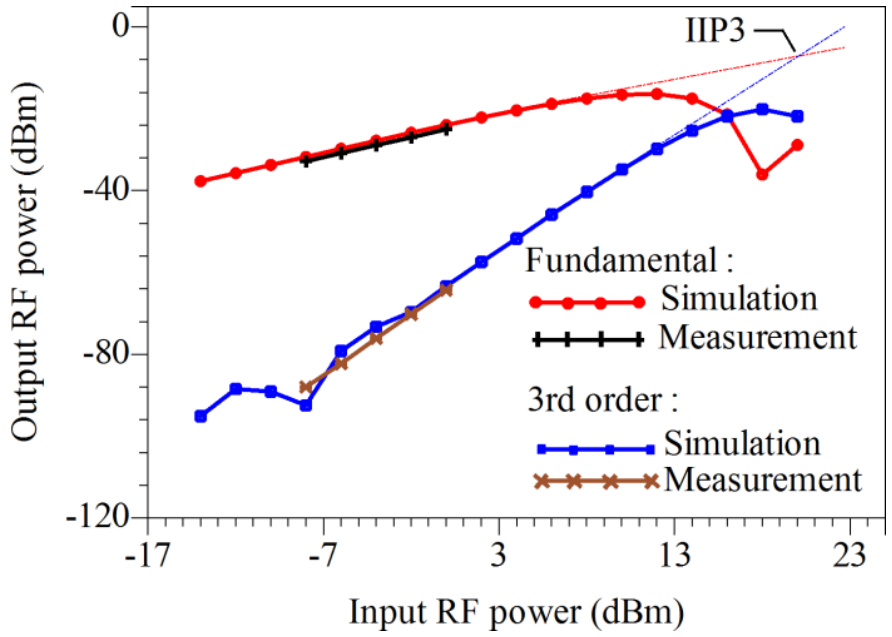

Fig. 6. Fundamental, third order tones and noise power density at $1 \mathrm{~Hz}$ bandwidth. RF tones at 2 and $2.05 \mathrm{GHz}$ and FSR is $6 \mathrm{GHz}$.

At a frequency of $3.3 \mathrm{GHz}$ (not shown here), the SFDR improves by $3 \mathrm{~dB}$ due to the increased and flatter gain response at this frequency.

The interferometric detection introduces an additional noise term induced by optical phase noise to intensity noise conversion. The noise term given by (7) represents this conversion effect and indicates a cosine dependence with RF frequency. This phase noise component depends on laser linewidth and drops to very low levels at RF frequencies near the FSR value. The simulation results of the link total noise power with and without laser phase noise for the modeled CW laser are shown in Fig. 7. This laser has a very narrow linewidth, typically lower than $10 \mathrm{kHz}$, and a RIN less than $-170 \mathrm{~dB} / \mathrm{Hz}$. The total noise follows the MZI response and is higher at gain peaks corresponding to half-FSR.

Although intensity modulated links exhibit lower noise power with highly stable monochromatic lasers and balanced photo-detection, phase-modulated links can attain similar noise level. Thus, better performance can be achieved with laser sources having lower phase noise and higher power. The PM $V_{\pi}$ and the MZI FSR also influence the link performance. Balanced photo-detection can provide $6 \mathrm{~dB}$ higher gain (see (4)) and reduce significantly the RIN noise level. Depending on the fronthaul network length, configurations can be optimized by choosing the best design parameter combination to improve the link performance.

Table II summarizes simulated link characteristics obtained with a high-power distributed feedback laser with an output optical power of $19 \mathrm{dBm}$, a linewidth of $10 \mathrm{kHz}$ (as before), a fiber length of $20 \mathrm{~km}$, an FSR of 6 and $10 \mathrm{GHz}$ and a carrier frequency matching exactly the half-FSR value The gain and noise values are given for the MZI operation at the quadrature point (at 3 and $5 \mathrm{GHz}$, respectively). The system linearity with an FSR of $6 \mathrm{GHz}$ is improved with a resulting SFDR of 110 $\mathrm{dB} . \mathrm{Hz}^{2 / 3}$. This represents an increase of $10 \mathrm{~dB}$ compared to the previous measured and simulated results. In addition, the noise level is reduced by $3 \mathrm{~dB}$ to around $-160 \mathrm{dBm} / \mathrm{Hz}$ from -157 $\mathrm{dBm} / \mathrm{Hz}$. Even better results are obtained with an FSR of 10 $\mathrm{GHz}$ with a further $4 \mathrm{~dB}$ improvement in the SFDR. 
Table II also includes performance predictions for an IM-DD link for the same system parameters, so that performance comparisons for both types of architectures can be carried out. The predictions confirm the results obtained in [27]. Both architectures suffer from the same noise contributions but the PM link is further impaired by the phase-to-intensity conversion noise from the MZI (as expressed in (7), especially when the small-signal gain is at its maximum). Thus, for the same link parameters, the noise level in the PM link depends on laser linewidth and can be higher than the noise level in an IMDD link as shown in Table II. But, the SFDR of the PM link is higher due to the higher gain and OIP3 performance.

In general, reductions in the noise level of IM-DD links can be achieved by balanced photo-detection but only if the dominant noise source is RIN. However, for PM links, given the more dominant dependence of the noise level on linewidth, such improvements are habitually the case.

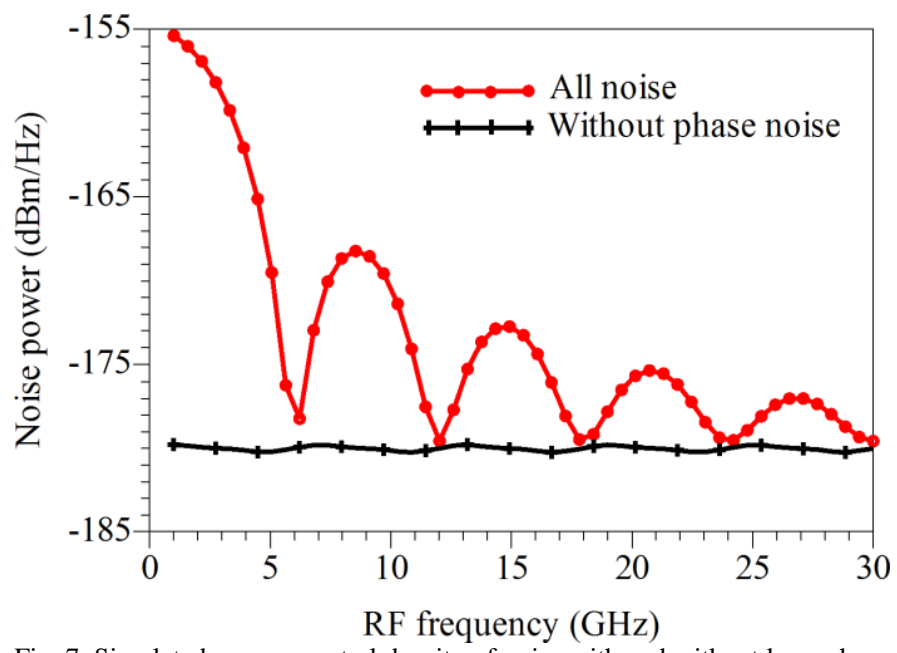

Fig. 7. Simulated power spectral density of noise with and without laser phase noise for an FSR of $6 \mathrm{GHz}$.

TABLE II

SUMMARIZED PREDICTED PERFORMANCES FOR 20 KM PM LINK AND COMPARISON TO IM-DD LINK

\begin{tabular}{lccc}
\hline \hline & $\begin{array}{c}\text { FSR=6 GHz } \\
\left(f_{\mathrm{RF}}=3 \mathrm{GHz}\right)\end{array}$ & $\begin{array}{l}\mathrm{FSR}=10 \mathrm{GHz} \\
\left(\mathrm{f}_{\mathrm{RF}}=5 \mathrm{GHz}\right)\end{array}$ & $\begin{array}{c}\mathrm{IM}-\mathrm{DD} \\
\left(\mathrm{f}_{\mathrm{RF}}=5 \mathrm{GHz}\right)\end{array}$ \\
\hline Gain $(\mathrm{dB})$ & -13.7 & -10.6 & -17.15 \\
IIP1 $(\mathrm{dBm})$ & 12.2 & 13.24 & 19.56 \\
IIP3 $(\mathrm{dBm})$ & 21 & 19.85 & 23.84 \\
SFDR $\left(\mathrm{dB} . \mathrm{Hz}^{2 / 3}\right)$ & 110 & 114.4 & 113.78 \\
Noise Power & -159.8 & -163.1 & -175.3 \\
$(\mathrm{dBm} / \mathrm{Hz})$ & & & \\
\hline \hline
\end{tabular}

\section{TRANSMiSSION OF 5G-TYPE WAVEFORMS OVER PM ROF LINKS}

The 5G New Radio (NR) will continue to employ the traditional cyclic prefix-orthogonal frequency division multiplexing (CP-OFDM) as the physical layer transmission scheme for both uplink and downlink. The Discrete Fourier Transform-OFDM (DFT-OFDM) will be considered for uplink modulation in some specific scenarios [2]. New numerologies, dedicated to $5 \mathrm{G}$ applications, have been defined to achieve higher data rates and optimal spectrum allocation. Currently, the 5G NR allows four different subcarrier spacings specified at multiples of $15 \mathrm{kHz}$ for data transmission (additional subcarrier spacings are expected in future releases) [2]. Besides the sub- $6 \mathrm{GHz}$ band, millimeter-wave bands (for example at 28 $\mathrm{GHz}$ and $60 \mathrm{GHz}$ ) have either already been planned for deployment or are being proposed for use in $5 \mathrm{G}$ access for ultradense area coverage [1]. Furthermore, to improve performance across a wide range of system metrics, several filtered variants of OFDM (F-OFDM) have been proposed, although at the expense of increased complexity [28].

In subsection IV.A, we present a comparison between experimentally measured and simulated (by employing the matched model described in Section III) Error Vector Magnitude (EVM) and dynamic range for single channel transmission for both CP-OFDM and F-OFDM. In addition, we show the potential for improved dynamic range by employing higher performance photonic components (using the optical link with predicted analog performances as shown in Table II). In subsection IV.B, a similar comparison is carried out for multi-channel transmission of CP-OFDM signals, followed by a simulation-based performance prediction using the matched model for larger aggregate bandwidth multiplexes.

\section{A. Single-channel transmission}

The setup shown in Fig. 8 was used to evaluate the performance of the phase-modulated RoF link for the two 5GOFDM-type waveforms with a 64-QAM modulation scheme. The baseband signal creation takes place in MATLAB and includes the generation of frequency-domain QAM samples, pilot insertion for tracking the channel frequency response, Inverse-Fast Fourier Transform (IFFT), Cyclic Prefix (CP) insertion, and the shaping filter (for the F-OFDM experiments). The time-domain In-phase and Quadrature (I/Q) sampled signal is then downloaded into an Arbitrary Waveform Generator (AWG), which performs digital-to-analog conversion and upconversion to an RF frequency of $2 \mathrm{GHz}$. The AWG-generated signal, through an input power control block (not shown) is then applied to the PM RF input and the modulated optical signal is transmitted over the amplified (through an EDFA) short-length fiber link (1 meter). The photo-detected signal is amplified and captured with a Tektronix 72304DX oscilloscope. The captured signal is processed offline in MATLAB with time-correction, filtering (for the F-OFDM experiments), FFT, frequencydomain equalization of the channel frequency response and demodulation, followed by EVM estimation. Table III summarizes the signal parameters, which have been matched for both measurements and simulations. Note that for the simulation results, the EDFA, AWG and oscilloscope noises have been modeled with noise sources matched to measured noise levels. Furthermore, the bandwidth of all measurement devices (AWG and oscilloscope) is high enough and can accommodate the employed signal bandwidths and RF frequencies, without performance degradation.

The measured EVM versus input RF power is compared to simulation results, obtained by using the model described in Section III, for both CP-OFDM and F-OFDM waveforms in 
Fig. 9. For CP-OFDM, the measured input power range (dynamic range) with respect to the 3GPP EVM specification of $8 \%$ in the case of 64-QAM [29], is about $23 \mathrm{~dB}$ (Fig. 9(a)). Note that although the system described here is for the uplink section of the fronthaul, the EVM results are compared to the more stringent $8 \%$ transmit 3GPP EVM specification. In a practical implementation, signal performance is expected to be degraded by wireless transmission, thus the more stringent transmit requirement ensures that some leeway for performance degradation is available.

The back-to-back (i.e. without an optical link) EVM is approximately $2.5 \%$ at an RF input power of $-10 \mathrm{dBm}$ for the CP-OFDM signal. The noise contribution from the AWG was modelled in simulation by incorporating a noise source at the $\mathrm{RF}$ input of the phase modulator to obtain a good agreement between simulation and measurement. For the F-OFDM signal, the measured and simulated EVM results are shown in Fig. 9(b), where the measured dynamic range is $22.5 \mathrm{~dB}$.

With an MZI-FSR of $10 \mathrm{GHz}$, the measurement results at an RF frequency of $2 \mathrm{GHz}$ show similar EVM behavior. The measured EVMs versus input RF power for a CP-OFDM and an F-OFDM signal are shown in Fig. 10. The measured dynamic range is about $20 \mathrm{~dB}$ for this FSR compared to $23 \mathrm{~dB}$ for an FSR of $6 \mathrm{GHz}$. This is a result of the lower link gain at the frequency of $2 \mathrm{GHz}$ for an FSR of $10 \mathrm{GHz}$ (where the gain peaks at $5 \mathrm{GHz}$ ).

For an FSR of $10 \mathrm{GHz}$, it is interesting to analyze the EVM performance when the transmitted signal is centered at $5 \mathrm{GHz}$ (the half-FSR point).

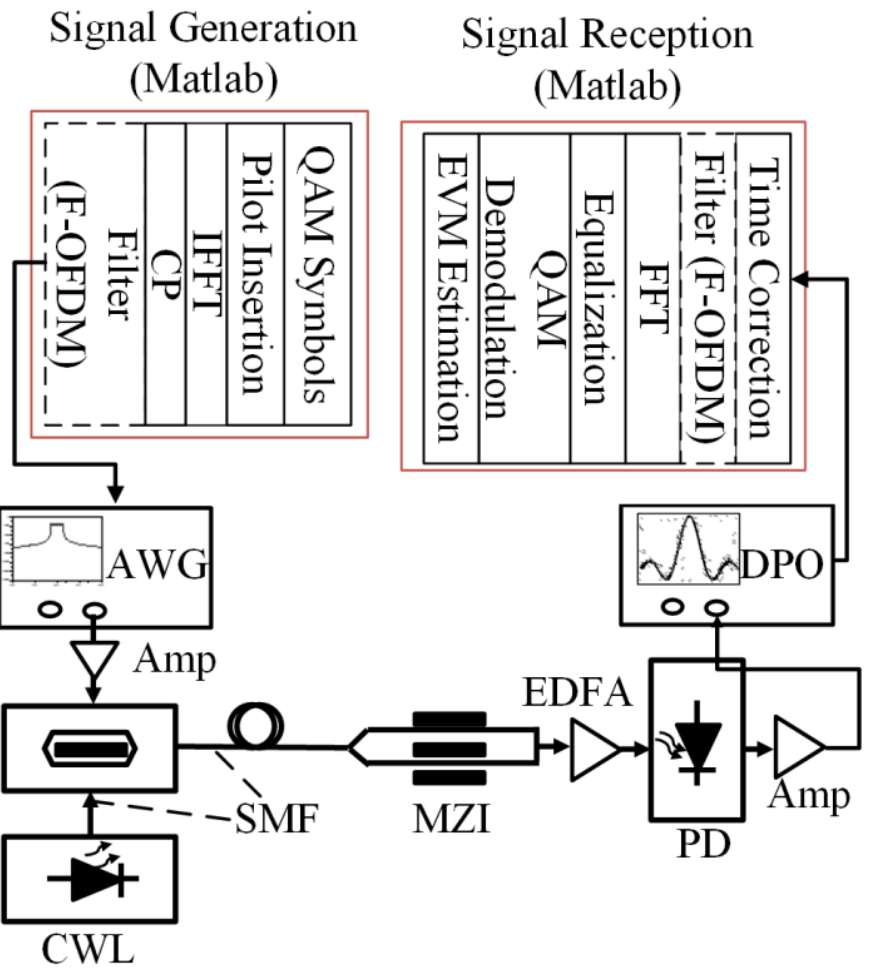

Fig. 8. Experimental setup for EVM measurements. Up-conversion to RF is carried out directly by the AWG. AWG, Arbitrary Waveform Generator; DPO, Digital Phosphor Oscilloscope; AMP, Amplifier; SMF, Single-Mode Fiber.
TABLE III

WAVEFORM PARAMETERS

\begin{tabular}{lc}
\hline \multicolumn{1}{c}{ Parameter } & value \\
\hline Subcarrier spacing (kHz) & 120 \\
IFFT/FFT size & 2048 \\
Num. pilots & 121 \\
Pilot separation (subcarriers) & 10 \\
Modulation & $64-\mathrm{QAM}$ \\
Data subcarriers & 1200 \\
Filter length (F-OFDM) & 1024 \\
PAPR (dB) & 10.7 \\
Bandwidth (MHz) & 144 \\
Data rate (Mbps) & 777 \\
\hline \hline
\end{tabular}
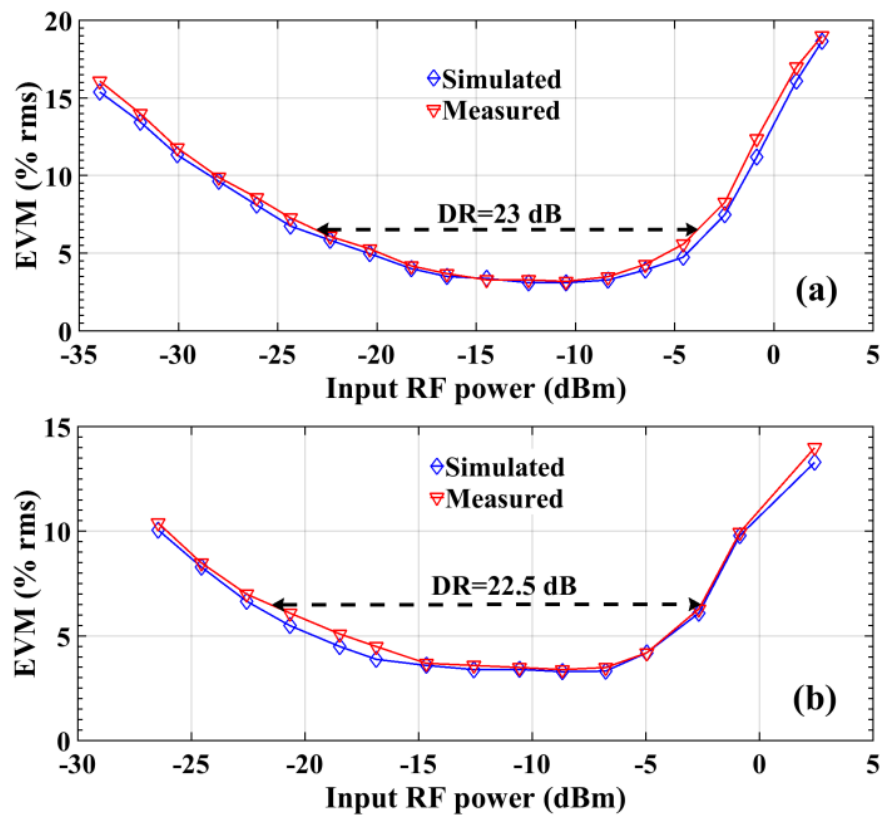

Fig. 9. Measured and simulated EVM versus input RF power for (a) CP-OFDM and (b) F-OFDM signal. RF frequency is $2 \mathrm{GHz}$ and FSR is $6 \mathrm{GHz}$.

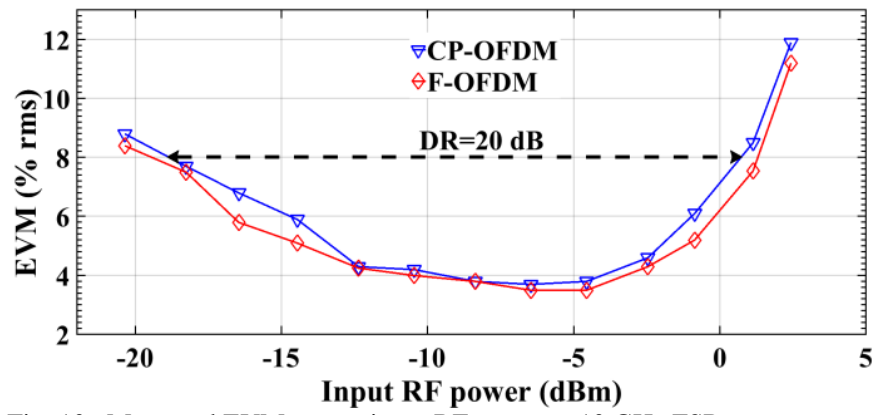

Fig. 10. Measured EVM versus input RF power at $10 \mathrm{GHz}$ FSR.

With the analog parameters given in Table II, the simulated EVM is shown in Fig. 11; the dynamic range is increased to 36 $\mathrm{dB}$, confirming that the choice of RF frequency and FSR value has a major effect on performance, as the link gain is maximized at the half-FSR point while the noise contribution is reduced with higher FSR (compared to lower FSR values).

\section{B. Multi-channel transmission}

The set-up for the experimental and simulation-based measurements used to evaluate the performance of the phase modulated RoF link with multi-channel signal transmission is 


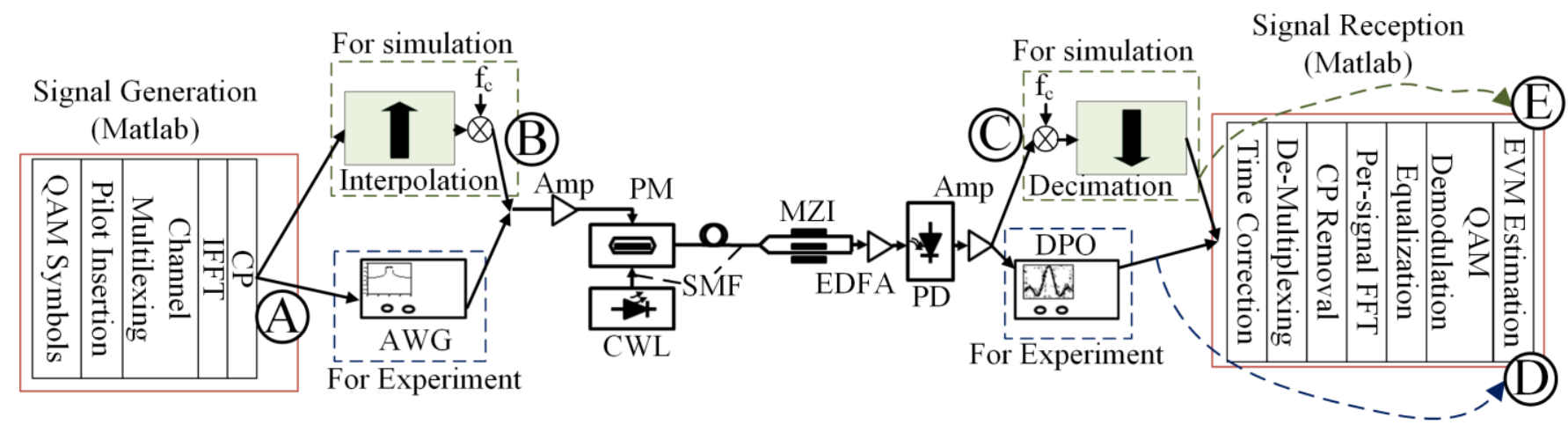

Fig. 12. Measurement and simulation set-up for multi-channel transmission. A: Spectrum view of input multiplexes; B: Spectrum view of input to optical link (simulation); C: Spectrum view of output from optical link (simulation); D: Measured EVM performance; E: Simulated-modeled EVM performance.

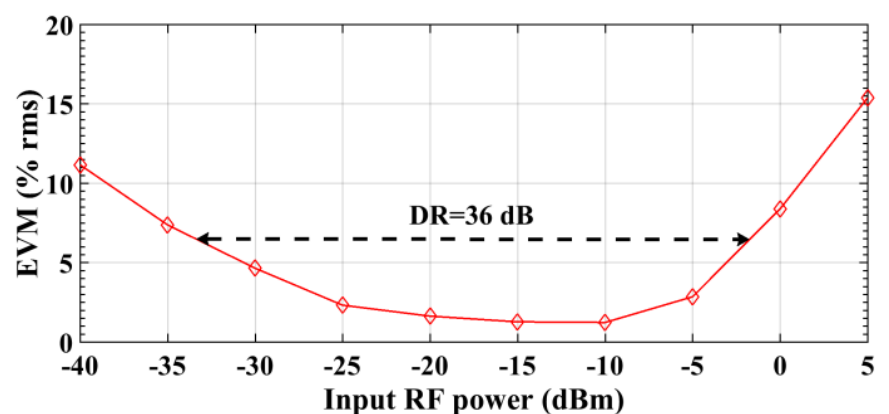

Fig. 11. Simulation-based prediction of EVM versus RF input power for the FOFDM signal. RF frequency is $5 \mathrm{GHz}, \mathrm{FSR}$ is $10 \mathrm{GHz}$, a CW laser with higher optical power and $20 \mathrm{~km}$ fiber length are assumed (see corresponding predicted analog performances in Table II).

shown in Fig. 12. The multiplex creation is carried out in MATLAB through a process similar to that used for the singlechannel experiments described in subsection III.A.

The multiple channels are multiplexed in the frequencydomain and simultaneously transformed into the time-domain through the IFFT. For the experimental measurements, the AWG is used to perform Single-Sideband (SSB) up-conversion of the multiplex to RF. For the simulation measurements, the AWG is represented by an interpolation block followed by a SSB up-converter that places the multiplex at the corresponding RF frequency. At the receiver side, following time correction, the channels are de-multiplexed using a digital filter bank, the $\mathrm{CP}$ for each channel is removed, and per-channel FFT and frequency-domain equalization are performed. Finally, following demodulation, the EVM performance for each channel is estimated. A more detailed description of the multiplexing and de-multiplexing processes can be found in [10].

A summary of the parameters used in the experimental and simulation set-ups is presented in Table IV. A first set of experimental and simulation-based measurements and their comparison is carried out at an FSR of $6 \mathrm{GHz}$, with a multiplex of 11 channels up-converted to an RF frequency of $1.6 \mathrm{GHz}$. Each channel has a subcarrier spacing of $60 \mathrm{kHz}$, and a bandwidth of $72 \mathrm{MHz}$ resulting in a total aggregate bandwidth (including pilot subcarriers) of $792 \mathrm{MHz}$. The specific size and bandwidth of the multiplex are chosen based on the bandwidth capabilities of the AWG. Then a simulation-based prediction
TABLE IV

MULTI-CHANNEL EXPERIMENT AND SiMULATION PARAMETERS

\begin{tabular}{lcc}
\hline \hline \multicolumn{1}{c}{ Parameter } & $\begin{array}{c}\text { 11-channel } \\
\text { multiplex }\end{array}$ & $\begin{array}{c}\text { 16-Channel } \\
\text { multiplex } \\
\text { (simulation) }\end{array}$ \\
\hline Sub-carrier spacing (kHz) & 60 & 120 \\
IFFT size & 2048 & 2048 \\
Num. of frames & 5 & 5 \\
Modulation & QAM-64 & QAM-64 \\
Carrier Frequency (GHz) & 1.6 & $3.1 / 3.5$ \\
FSR (GHz) & 6 & 10 \\
Data subcarriers & 1200 & 1200 \\
Per-channel bandwidth (MHz) & 72 & 144 \\
Aggregate data rate (Gbps) & 4.3 & 12.4 \\
\hline \hline
\end{tabular}

with a larger aggregate bandwidth, 16-channel multiplex is carried out for an FSR of $10 \mathrm{GHz}$, with the multiplex upconverted to an RF frequency of $3.1 \mathrm{GHz}$. Each channel has a subcarrier spacing of $120 \mathrm{kHz}$ and a bandwidth of $144 \mathrm{MHz}$ resulting in a total aggregate bandwidth (including pilot subcarriers) of $2.3 \mathrm{GHz}$. Finally, an additional simulation-based prediction is carried out for the same 16-channel multiplex, upconverted to an RF frequency of $3.5 \mathrm{GHz}$ and employing the optical link with higher performance photonic components and an optical fiber span of $20 \mathrm{~km}$ (see the corresponding analog performance predictions for this link in Table II).

Fig. 13 shows the input spectra (point A) for the 11-channel and 16-channel multiplexes as produced in MATLAB. Note that the multiplex bandwidth is higher than the aforementioned aggregate bandwidth due to the inclusion of null subcarriers/frequency guard bands. These guard bands lead to a reduction in the effective spectral efficiency, but their size can be reduced through optimization of the filtering performed in the receiver de-multiplexing process. However, such optimization is not in the scope of the work presented here.

Figures 14 to 16 show the input and output to/from optical link spectra (points B and C) and the respective EVM (as a \% of the rms constellation value) per channel results (points $\mathrm{E}$ and D). Note that best fit traces (dotted traces) that represent average trend behavior are used for all EVM results to aid the visualization of how (on average) the EVM performance changes with frequency. 

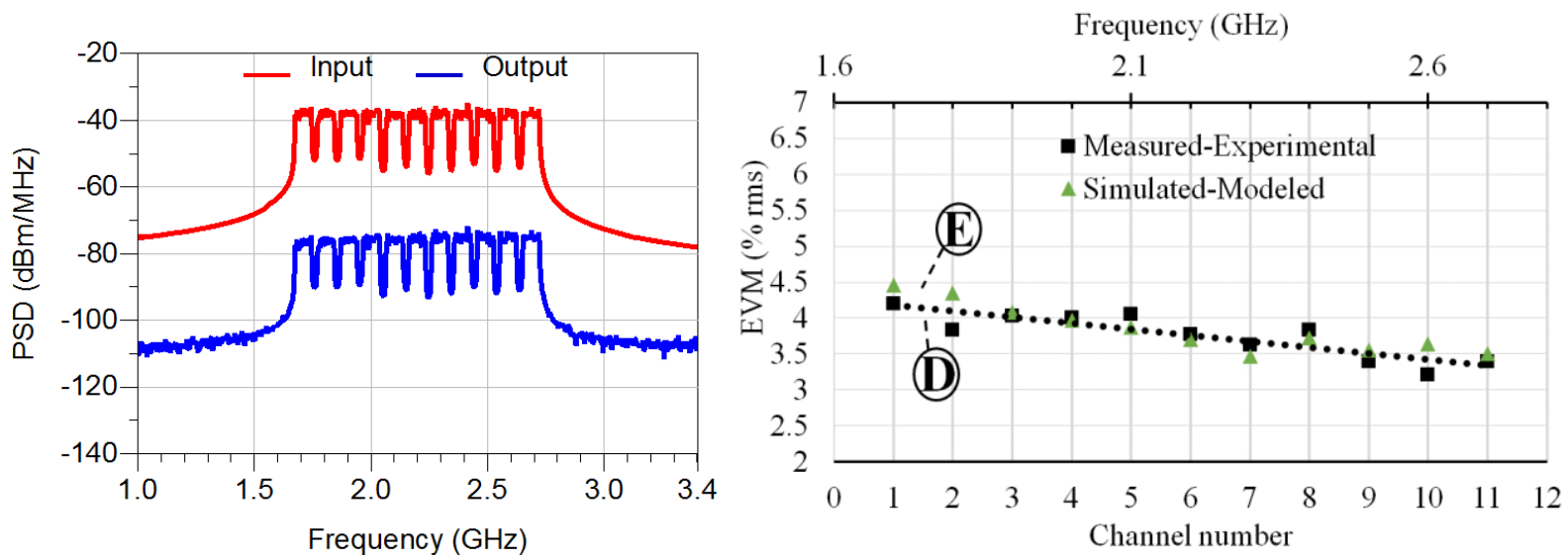

Fig. 14. (Left) Spectrum view of input (point B in Fig. 14) and output (point $\mathrm{C}$ in Fig. 12) from optical link for the 11-channel multiplex with FSR=6 GHz and $\mathrm{f}_{\mathrm{c}}=1.6 \mathrm{GHz}$ (simulation). (Right) Measured-experimental and simulated-modeled EVM performance (points D and E respectively in Fig. 12).
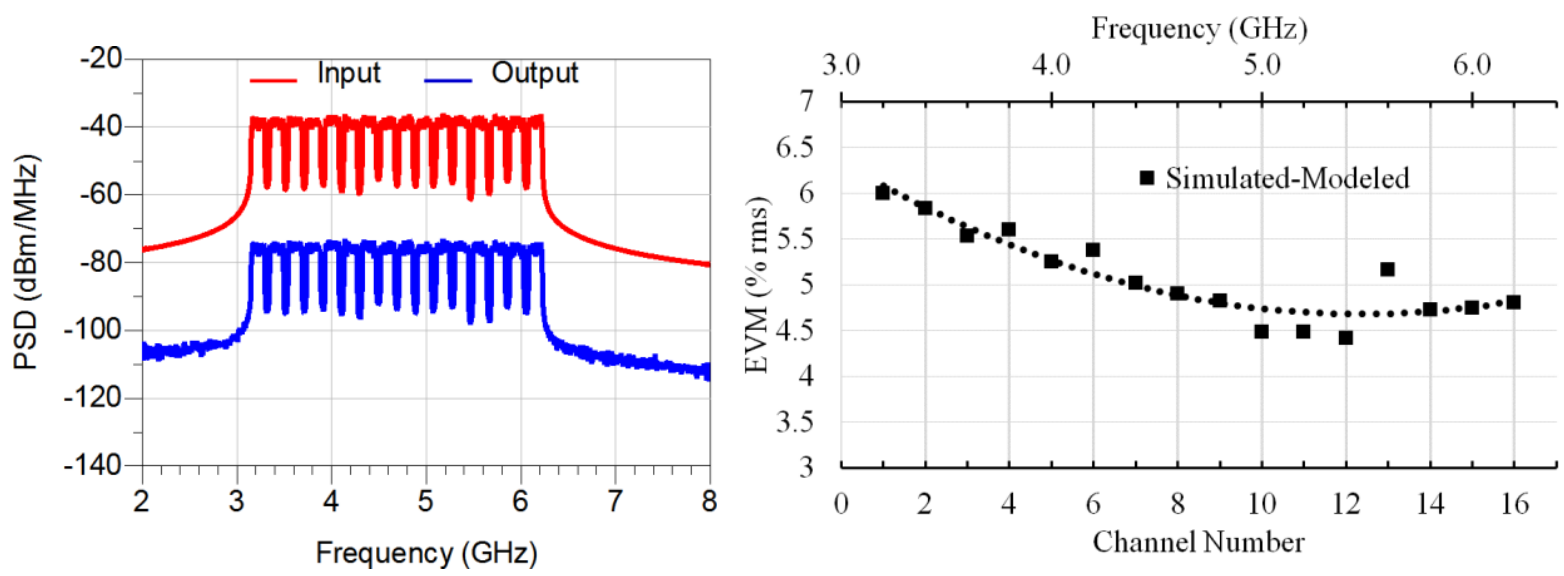

Fig. 15. (Left) Spectrum view of input (point B in Fig. 12) and output (point $\mathrm{C}$ in Fig. 12) from optical link for the 16-channel multiplex with FSR=10 GHz and $\mathrm{f}_{\mathrm{c}}=3.1 \mathrm{GHz}$ (simulation). (Right) Simulated-modeled EVM performance (point E in Fig. 12).
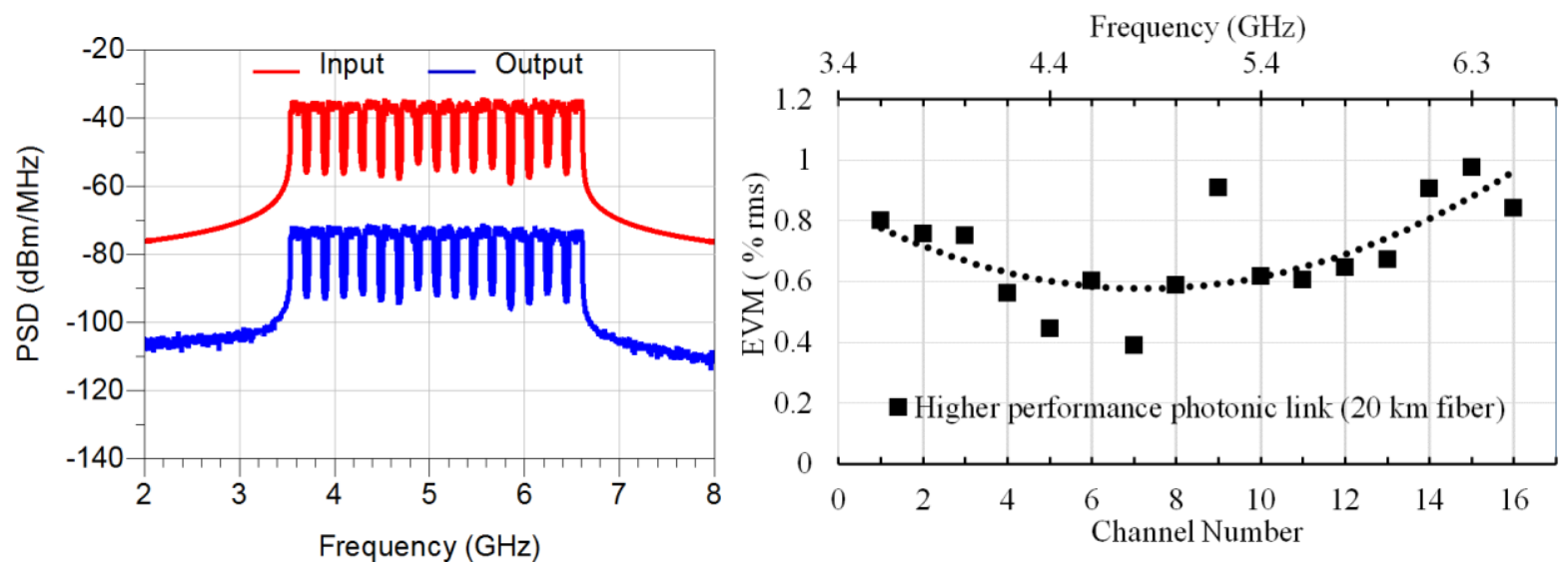

Fig. 16. (Left) Spectrum view of input (point B in Fig. 12) and output (point $C$ in Fig. 12) from optical) for the 16-channel multiplex with the higher performance optical link and $20 \mathrm{~km}$ fiber span, with $\mathrm{FSR}=10 \mathrm{GHz}$ and $\mathrm{f}_{\mathrm{c}}=3.5 \mathrm{GHz}$ (simulation). (Right) Simulated-modeled EVM performance (point $\mathrm{E}$ in Fig. 12).

In Fig. 14, for the 11-channel multiplex, the comparison between measured and simulated-modeled EVM results shows a good match across all the channels, corresponding to a multiplex bandwidth (including the frequency guard bands inbetween the channels) of more than $1 \mathrm{GHz}$. EVM values for all channels are well within 3GPP specifications for 64-QAM [29].
The resulting aggregate user data rate is approximately 4.3 Gbps. The EVM traces show a reduction in EVM (corresponding to an SNR increase) as expected for channels closer to the FSR gain peak (the half-FSR point at $3 \mathrm{GHz}$ ), due to the increased RF gain around this region (see Fig. 4). 
(a)

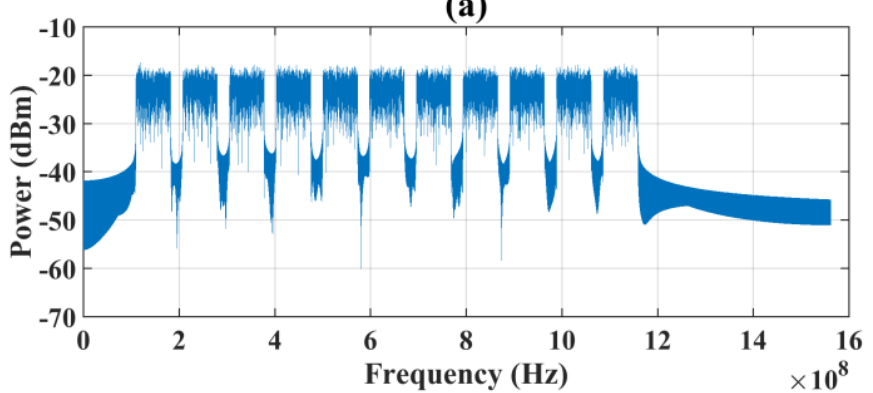

(b)

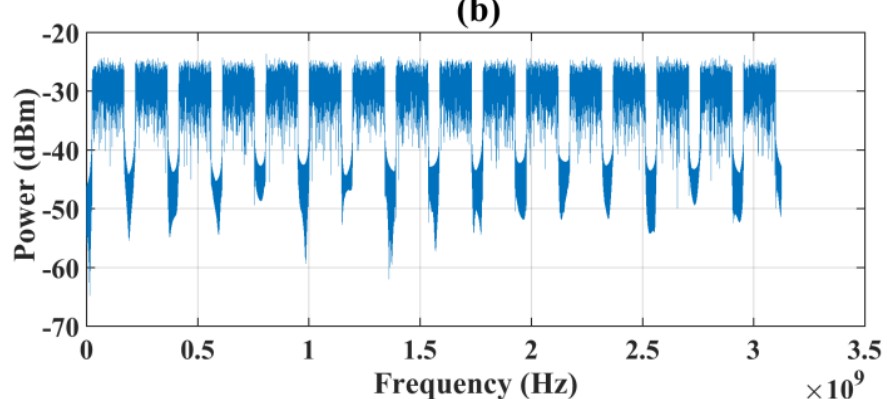

Fig. 13. Spectrum view of input multiplexes at point A in Fig. 12. For (a) 11channel multiplex and (b) 16-channel multiplex.

In Fig. 15, the simulation-based performance prediction for the 16-channel multiplex shows promising EVM performance, well within 3GPP limits for 64-QAM modulation. The multiplex now has an aggregate user data rate in excess of 12.4 Gbps, and due to the larger multiplex bandwidth, the EVM trace follows the trend of a larger portion of the FSR gain curve. It thus shows the expected dip (corresponding to the highest SNR) close to the FSR gain peak at 5GHz. Due to the position of the multiplex in frequency, more of the gain curve on the left side of the gain peak (frequencies from 0 to $5 \mathrm{GHz}$ ), corresponding to channels 1 to 10 , is followed.

In Fig. 16, the simulation-based performance prediction for the 16-channel multiplex with the optical link with higher performance photonic components and a fiber span of $20 \mathrm{~km}$ shows very good EVM performance across all channels as a result of the higher RF gain of the link (a result of the increase in the input optical power from the $\mathrm{CW}$ laser). The multiplex is now centered approximately at the half-FSR gain peak point at $5 \mathrm{GHz}$. As a result the average EVM trend (dotted line) shows a dip approximately at the half-FSR point which corresponds to channel 8, with channels further from this point showing progressively worse performance, as expected. Thus, in general, there is clear potential for optimization in performance, by appropriate RF frequency placement of multiplexes in accordance with the FSR employed by the PM, while larger FSR values are beneficial for larger aggregate bandwidth multiplexes.

\section{CONCLUSION}

A combined intensity and phase modulated radio over fiber link has been proposed for efficient $5 \mathrm{G}$ fronthauling. The uplink section employs an un-biased phase modulator that can be fed from a centrally distributed laser source. As a result, RU complexity and power consumption are reduced. The experimental characterizations of the phase-modulation and interferometric detection link have shown promising analog performance, demonstrating the link's ability to support the transmission of 5G-type waveforms. Experimentally measured EVM results for wide bandwidth single-channel and multichannel transmission of 5G-type signals with an aggregate data rate of $4.3 \mathrm{Gbps}$ are well within 3GPP EVM specifications for 64-QAM modulation, over a wide dynamic range. A simulation setup based on electrical circuit modelling of microwavephotonic components with specific consideration of optical field modelling as a circuit current, has been used to model the proposed system. Very good agreement between measured and modelled performances has been shown, which can be used for further system optimization and prediction. Such simulationbased predictions for multi-channel transmission of 16 channels and a total aggregate data rate of 12.4 Gbps show promising performance, well within 3GPP EVM requirements for 64QAM. The potential for improved performance, by employing higher performance photonic components, is also demonstrated by simulation.

\section{REFERENCES}

[1] ITU, "Setting the Scene for 5G: Opportunities \& Challenges," ITU report, 2018 [Online]. Available: https://www.itu.int/en/ITUD/Documents/ITU_5G_REPORT-2018.pdf. Accessed on: Apr. 08, 2019.

[2] Release 15 Description; Summary of Rel-15 Work Items (Release 15), 3GPP Technical Specification Group Services and System Aspects, TR 21.915, V1.0.0, Mar. 2019 [Online]. Available: https://www.3gpp.org/DynaReport/status-report.htm. Accessed on: Apr. 08, 2019.

[3] Study on New Radio Access Technology: Radio Access Architecture and Interfaces (Release 14), 3GPP Technical Specification Group Radio Access Network, TR 38.801, V14.0.0, Mar. 2017 [Online]. Available: http://www.3gpp.org/DynaReport/38-series.htm. Accessed on: Apr. 08, 2019.

[4] Common Public Radio Interface (CPRI); Interface Specification, CPRI Specification V7.0, Oct. 2015 [Online]. Available: http://www.cpri.info/spec.html. Accessed on: Apr. 08, 2019.

[5] N. Gomes et al., "Boosting 5G Through Ethernet: How Evolved Fronthaul Can Take Next-Generation Mobile to the Next Level," IEEE Veh. Technol. Mag., vol. 13, no. 1, pp. 74-84, Mar. 2018.

[6] N. J. Gomes, P. Assimakopoulos, M. K. Al-Hares, U. Habib, S. Noor "The New Flexible Mobile Fronthaul: Digital or Analog, or Both?" in Proc. Int. Conf. on Transparent Optical Networks (ICTON), Trento, Italy, 2016, pp. $1-4$.

[7] C. Ranaweera, E. Wong, A. Nirmalathas, C. Jayasundara and C. Lim, "5G C-RAN with Optical Fronthaul: An Analysis from a Deployment Perspective," J. Lightw. Technol., vol. 36, no. 11, pp. 2059-2068, Dec. 2018.

[8] N. J. Gomes and P. Assimakopoulos, "Optical Fronthaul Options for Meeting 5G Requirements," in Proc. Int. Conf. on Transparent Optical Networks (ICTON), Bucharest, Romania, 2018, pp. 1-4.

[9] Y. Yang, C. Lim and A. Nirmalathas, "Investigation on Transport Schemes for Efficient High-Frequency Broadband OFDM Transmission in Fibre-Wireless Links," J. Lightw. Technol., vol. 32, no. 2, pp. 267-274, Jan. 2014.

[10] S. Noor, P. Assimakopoulos, N. J. Gomes, "A Flexible Subcarrier Multiplexing System with Analog Transport and Digital Processing for 5G (and beyond) Fronthaul," J. Lightw. Technol., vol. 37, no. 14, pp. 3689-3700, 15 Jul. 2019.

[11] C. Browning, A. Delmade, Y. Lin, D. Geuzebroek, and L. Barry, "Optical Heterodyne Millimeter-Wave Analog Radio-over-Fiber with Photonic Integrated Tunable Lasers," in Proc. Opt. Fiber Commun. Conf., (OFC), San Diego, CA, USA, 2019, paper W1I.4.

[12] M. Sung, S.-H. Cho, J. Kim, J. K. Lee, J. H. Lee and H. S. Chung, "Demonstration of IFoF-Based Mobile Fronthaul in 5G Prototype With 
28-GHz Millimeter Wave,”. Lightw. Technol., vol. 36, no. 2, pp. 601609, Jan. 2018.

[13] U. Habib, A. E. Aighobahi, T. Quinlan, S. D. Walker and N. J. Gomes, "Analog Radio-Over-Fiber Supported Increased RAU Spacing for 60 GHz Distributed MIMO Employing Spatial Diversity and Multiplexing," J. Lightw. Technol., vol. 36, no. 19, pp. 4354-4360, Oct. 2018.

[14] D. Wake et al., "A Comparison of Radio Over Fiber Link Types for the Support of Wideband Radio Channels," J. Lightw. Technol., vol. 28, no. 16, pp. 2416-2422, Aug. 2010.

[15] C. P. Liu and A. Seeds, "Transmission of Wireless MIMO-Type Signals Over a Single Optical Fiber Without WDM," IEEE Trans. Microw. Theory Tech., vol. 58, no. 11, pp. 3094-3102, 2010.

[16] X. Liu, H. Zeng, N. Chard, F. Effenberger, "Efficient Mobile Fronthaul via DSP-Based Channel Aggregation," J. Lightw. Technol., Vol. 34, No. 6, pp. 1556-1564, 2016.

[17] N. Argyris, et al.," "A 5G mmWave Fiber-Wireless IFoF Analog Mobile Fronthaul link with up to $24 \mathrm{~Gb} / \mathrm{s}$ Multi-band Wireless Capacity," $J$. Lightw. Technol, vol. 37, no. 12, pp. 2883-2891, Jun. 2019.

[18] S. A. Khwandah, J. P. Cosmas, I. A. Glover, P. I. Lazaridis, N. R. Prasad and Z. D. Zaharis, "Direct and External Intensity Modulation in OFDM RoF Links," IEEE J. Photonics, vol. 7, no. 4, pp. 1-10, Jul. 2015.

[19] V. J. Urick, F. Bucholtz, P. S. Devgan, J. D. McKinney and K. J. Williams, "Phase Modulation with Interferometric Detection as an Alternative to Intensity Modulation With Direct Detection for AnalogPhotonic Links," IEEE Trans. Microw. Theory Tech., vol. 55, no. 9, pp. 1978-1985, Sep. 2007.

[20] A. Caballero, D. Zibar and I. T. Monroy, "Performance Evaluation of Digital Coherent Receivers for Phase-Modulated Radio-Over-Fiber Links," J. Lightw. Technol., vol. 29, no. 21, pp. 3282-3292, Nov. 2011.

[21] V. R. Pagán and T. E. Murphy, "Phase-modulated radio-over-fiber systems," in Proc. Opt. Fiber Commun. Conf. and Exposition and the Nat. Fiber Optic Engineers Conf. (OFC/NFOEC), Anaheim, CA, 2013, pp. 13.

[22] Z. Yang, Q. Yu, J. Zang, J. C. Campbell and A. Beling, "Phase-Modulated Analog Photonic Link With a High-Power High-Linearity Photodiode," J. Lightw. Technol., vol. 36, no. 18, pp. 3805-3814, Sept. 2018.

[23] K. Kitayama, "Digital coherent technology for optical fiber and radioover-fiber transmission systems," in Proc. IEEE Int. Topical Meeting on Microwave Photonics (MWP), Alexandria, VA, 2013, pp. 1-4.

[24] A. Billabert, A. Kabalan, S. Faci, R. Zakaria, M. Moutaly and C. Algani, "Simulation approach of wireless OFDM and FBMC signals transmission over fiber based on equivalent electrical models," in Proc. 19th Int. Conf. on Transparent Optical Networks (ICTON), Girona, Spain, 2017, pp. 1-4.

[25] W.E Kassa, S. Faci, A-L. Billabert et al., "Circuit modelling of phase modulated microwave optical links and performance analysis," Opt. Quant. Electron (2017) 49: 394.

[26] V. J. Urick, Keith J. Williams, Jason D. McKinney, "External phase modulation with interferometric detection," in Fundamentals of Microwave Photonics, John Wiley \& Sons, 2015, ch. 7, pp. 273-311.

[27] M. Emmaeinna, S. Faci, A. Billabert, A. Kabalan, C. Algani and M. L. Diakité, "Performance Analysis of Radio-over-Fiber Based on PhaseModulation and Direct-Detection for the Future 5G Network," Proc. Int. Conf. on Transparent Optical Networks (ICTON), Bucharest, Romania, 2018, pp. 1-4.

[28] Y. Liu et al., "Waveform Design for 5G Networks: Analysis and Comparison," IEEE Access, vol. 5, pp. 19282-19292, 2017.

[29] Base Station (BS) radio transmission and reception (Release 15), 3GPP Technical Specification Group Radio Access Network; NR, TS 38.104, V15.0.0, Dec. 2017 [Online]. Available: http://www.3gpp.org/DynaReport/38-series.htm. Accessed on: Apr. 08, 2019. 